\title{
Laser annealing of shape memory alloys: A versatile tool for developing smart micro-devices
}

\author{
Y. Bellouard, T. Lehnert ${ }^{1}$, R. Clavel, T. Sidler ${ }^{2}$ and R. Gotthardt ${ }^{1}$ \\ Institut de Systèmes Robotiques, DMT, École Polytechnique Fédérale de Lausanne, \\ 1015 Lausanne, Switzerland \\ 1 Institut de Génie Atomique, DP, École Polytechnique Fédérale de Lausanne, 1015 Lausanne, \\ Switzerland \\ ${ }^{2}$ Institut d'Optique Appliquée, DMT, École Polytechnique Fédérale de Lausanne, \\ 1015 Lausanne, Switzerland
}

\begin{abstract}
From a shape change point of view, the shape memory effect is not intrinsically reversible. Therefore developing Shape Memory Alloys (SMA) actuators, i.e. devices producing a motion, requires a suitable method to bypass this intrinsic property. Since the shape memory effect discovery, different methods, either intrinsic like the «Two-Way Shape Memory Effect » or extrinsic like the use of a biasing element have been investigated. In this paper, a new method based on a local annealing of the material is presented. A laser is used to locally crystallise amorphous thin film or re-crystallise cold-worked sheet of SMA material. For instance, this method allows the development of highly integrated micro-devices made out of one single piece of material. The basic principle, a typical setup and some effects of the laser annealing on the material properties are presented followed by some examples of monolithic micro-devices developed using this concept.
\end{abstract}

\section{INTRODUCTION}

From a shape change point of view, the shape memory effect is not intrinsically reversible. If no thermo-mechanical treatment is applied on the material, the thermally-induced martensite does not have a preferential orientation. Therefore, the specific volume occupied by the martensite is the same than the specific volume of the austenite. Getting around this non-reversibility of the shape change has been the « leimotiv » of many researches on shape memory actuators. Several solutions have been proposed at different levels of conception:

- At a material level, special fabrication processes (for instance melt-spinning) and postfabrication processes (Two-Way Shape Memory Effect) have been investigated. These methods having the common consequence of creating an internal stress within the material. This internal stress will favour a preferred orientation of martensite variants on cooling.

- At a design level, developments have focused on reversible effect induced by external means like for instance the use of a bias spring.

In this paper, a new method based on a local treatment of the material is presented. The basic idea is to apply locally the annealing step required in the fabrication of SMA devices made out from amorphous thin film as well as from cold-worked sheet. Indeed, a non homogeneous structure will be induced: annealed zones will have an active function while remaining zones will behave like a "passive» material. Such method allows to integrate different mechanical behaviors within one single piece of material. Moreover, in contrary of existing methods, it can be adapted to any kind of design. This new approach is motivated by applications of SMA in micro-engineering where integration is required to avoid tough assembly tasks and to reduce effects of adhesion forces at micro-scale. 


\section{LASER ANNEALING OF SHAPE MEMORY ALLOYS (LASMA)}

\subsection{Experimental setup}

The basic experimental setup is shown on figure 1. The laser source is given by fiber pigtailed laser diode with a maximum available optical power of $1.3 \mathrm{~W}$. The beam has a circular shape. Collimating and focusing lenses are chosen to have a one-to-one ratio. The spot diameter has been measured using the knife-edge method. The diameter is $100+/-10 \mu \mathrm{m}$. Therefore, the maximum optical power per surface unit i.e. the irradiance is about $40 \mathrm{~W} / \mathrm{mm}^{2}$. The spot position on the sample is given by a fast-steering two-axis scanner.

Thanks to a vision system, a spot path, a scanning speed and an optical power can be defined point-to-point and programmed using a computer interface. Several annealing strategies and profiles can therefore be defined depending on the geometry of the sample and the desired functionalities. To control the material state during the annealing process, two kinds of sensors are used: the first one uses a four-point resistive measurement while the second one is a Si-Ge nearinfrared sensor ( $1 \mu \mathrm{m}$ to $2.5 \mu \mathrm{m}$ wavelength).

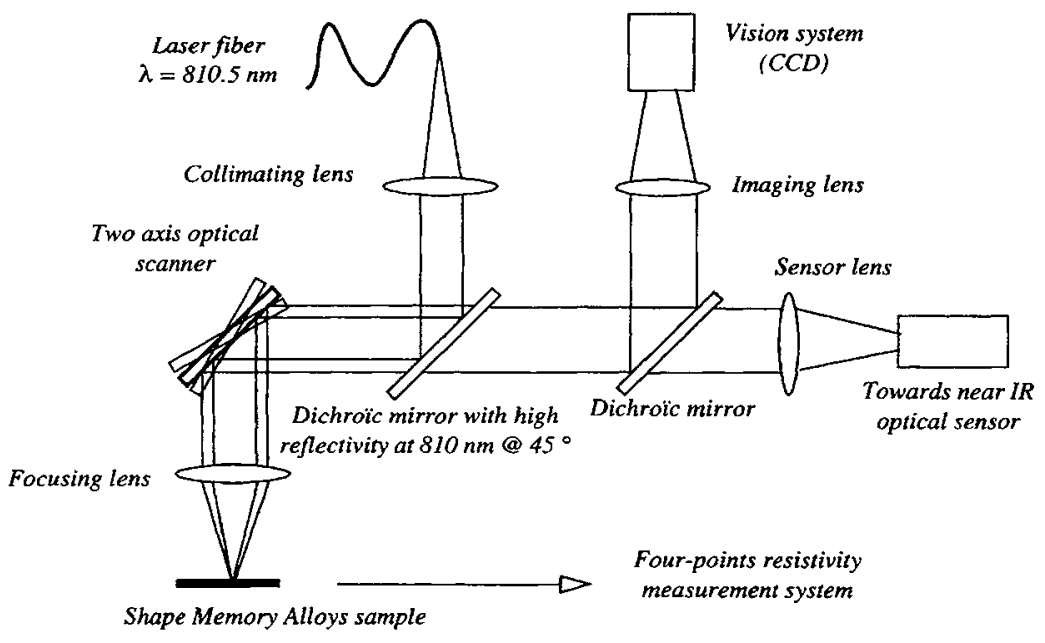

Figure 1: Laser annealing experimental setup

As the heat is localized in a very small area, measuring precisely the temperature is rather difficult: infrared cameras for instance, in addition to be expensive, are very sensitive to the surface state and to the sample's shape while thermocouples can hardly be used due to the small size of components. Therefore, a method based on multiple sensors measurements is preferred: resistance variation measurements give us an average information of the internal state of the material while the infrared sensor gives a mean temperature-like signal on a circular area for which the diameter is defined by the ratio between the focusing lens and the sensor lens. 


\subsection{Laser annealing of thin films}

The LASMA process was first applied on thin films materials fabricated by RF magnetron sputter deposition in a vacuum chamber. Several experiments have been performed in order to caracterize and to draw out some characteristics of the laser annealing. In a first one, the transformation behavior as it can be measured by differential scanning calorimetry was investigated. For that purpose, a square area of about $5 \times 5 \mathrm{~mm}^{2}$ from a bulk thin film has been annealed. The scanning strategy is shown on figure 2 . The path of the laser is chosen to avoid as much as possible an overlap between scanned lines and therefore to have the same heating condition for each zone of the scanned path. Typical parameters which need to be set carefully are the scanning speed, the distance between lines and obviously the irradiance. As it can easily be imagined, these parameters are closely related.
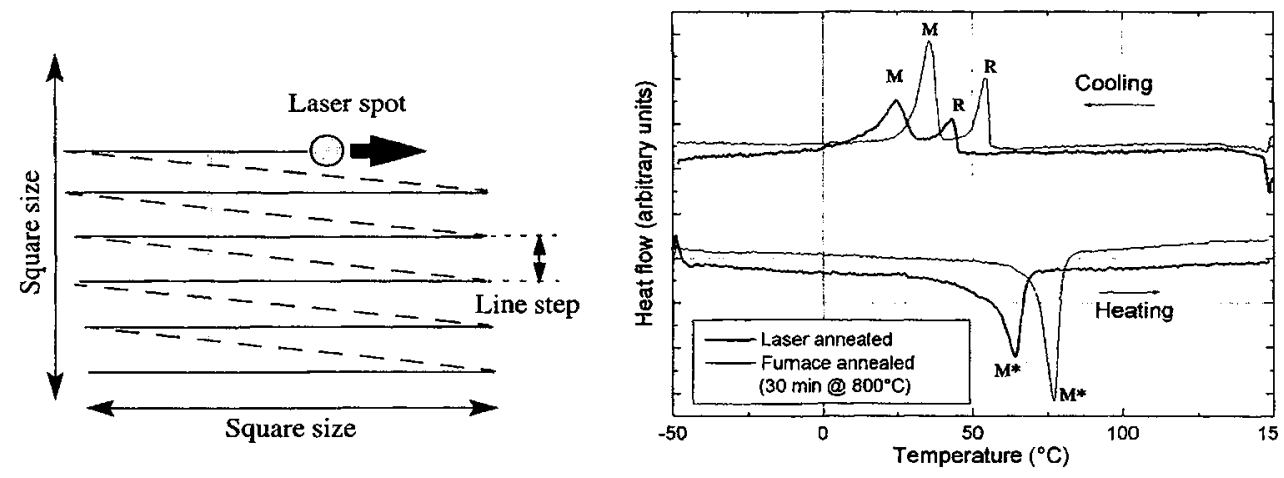

Figure 2: Scanning strategy for a square surface. The dotted lines represent trajectory where the laser beam is off. Critical parameters are the scanning speed related to optical power as well as the distance between each line.

Figure 3: DSC measurements of a thin film annealed by furnace and annealed by laser.

\section{Differentials Scanning Calorimetry investigations}

Differentials Scanning Calorimetry (DSC) curves are shown on figure 3: a laser annealed piece of thin film (thick curve) is compared with a piece of the same thin film annealed in a furnace. During heating one single peak $\left(\mathrm{M}^{*}\right)$ corresponding to the martensite to austenite transformation appears. On cooling, two peaks can be seen. The first one $(R)$ is identified as the Austenite to R-Phase transformation while the second one is related to R-Phase to martensite (M) transformation. In the case of the laser-annealed one, peaks are wider and smaller in height compared to those of the other sample. This difference might be explained by the less homogeneous temperature distribution occuring during the laser annealing treatment, resulting in an inhomogenous structure of the material. The hysteresis of the laser-annealed one is smaller. This might be due to the quasi-quenching effect which results of the rapid cooling of the affected zone.

\section{Strain induced by the laser annealing}

In these measurements, the strain behavior during the annealing process was investigated. For that purpose, a special setup was made: a laser diode with a rectangular-shape spot was used to anneal a structure consisting in two beams joined to each other with an angle of ninety degree (figure 4). The larger beam is locally annealed. Thanks to a cantilever placed at the beam's junction and the device geometry, any change in the length of the laser-affected beam is being amplified. The cantilever has also a heat sink function. 
This guarantee that the heat flow within the unaffected beam does not induce a temperature raised greater than $150^{\circ} \mathrm{C}$. For measuring the displacement of the cantilever's end, a computerbased micro-vision system is used. This technology allows a non-contact measurement within the plane.

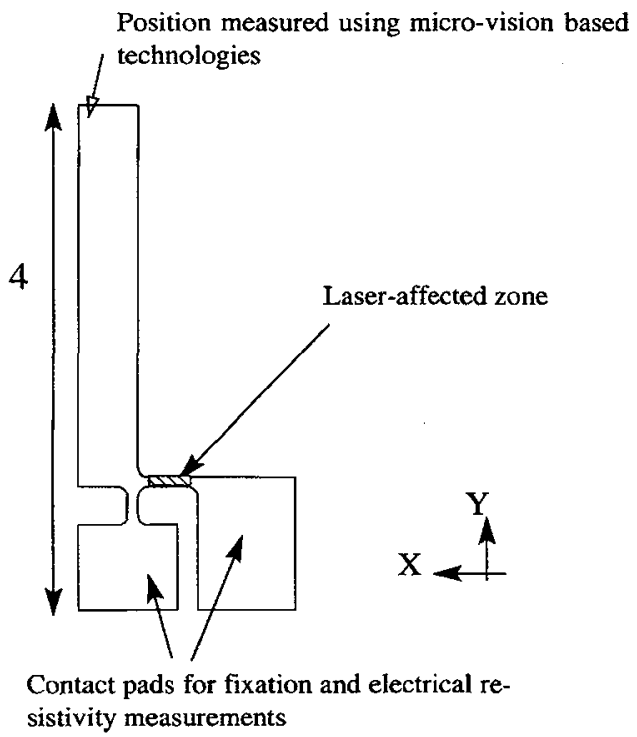

In this experiment the laser spot is $500 \mu \mathrm{m}$ long and $20 \mu \mathrm{m}$ wide. The irradiance was typically $70 \mathrm{~W} / \mathrm{mm}^{2}$. The laser-affected beam is $75 \mu \mathrm{m}$ wide and $450 \mu \mathrm{m}$ long. Therefore, the laser beam covers all the length of the cantilever. No special atmosphere was used during the experiment. In consequence, colors due to the oxydation are clearly visible. The temperature was controlled using an infrared camera. However, as mentionned before this method was not enough accurate. The scattering between two measurements is in the order of $+/-50^{\circ} \mathrm{C}$.

For various laser pulses and irradiation times, three different behaviours were observed:

- Fusion: the cantilever has been melted. This is observed for large power beam and $/$ or rather long irradiation time (typically more than $50 \mathrm{~ms}$ ).

- Contraction: the length of the beam has

Figure 4: Micro-cantilever for strain measurements

been reduced. A close inspection of the irradiated part shows that the material has been locally melted and rapidly solidified. Waves structures, out of the plane can be observed on the affected zone. This contraction occurs when the irradiation time is short (less than $20 \mathrm{~ms}$ ) and when the power is large.

- Expansion: the length of the beam have increased. The surface of the lsaer-annealed beam was still flat without any deformation. This was observed for laser pulse greater than $50 \mathrm{~ms}$ with a low power.

To furher investigate this expansion phenomenon, samples were submitted to a triangular laser power signal (figure 5).
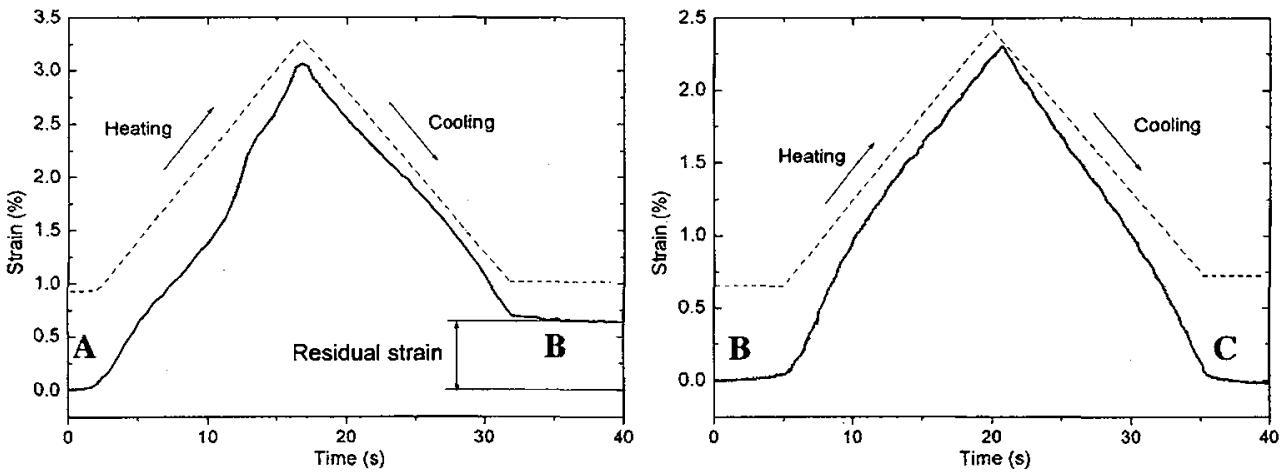

Figure 5: Relative strain behavior of the laser irradiated beam. A triangle signal of power is applied. The peak irradiance was about $70 \mathrm{~W} / \mathrm{mm}^{2}$. First and second cycles are shown (left and right curves). 
Curves on figure 5 represent the strain variation behaviour during a progressive increase and decrease of the beam power. This is a relative measurement of the cantilever's end motion. That means that the origin is set at the beginning of each experiment and corresponds to the initial position of the cantilever. Therefore, the chronological sequence is A-B-C on figure 5.

The strain increases with the increase of power which is characteristic of the thermal expansion of the material. However, the shape of the curve on heating is not the same than the shape on cooling down. The slope on heating is rather inhomogeneous and more important than the slope on cooling leading to a residual strain remaining at room temperature. If the same experiment is repeated once again, the maximum strain amplitude is smaller and no more residual strain is observed. The same experiment has been repeated several times leading to the same curves than observed in the second one.

A possible interpretation is the following: in the initial configuration ( $A$ on figure 5), the thin film is amorphous or partially amorphous ${ }^{1}$. When the temperature is enough high, the material crystallises which leads to a size reduction as the crystallised structure is more compact than the amorphous one. The transition between amorphous to crystallised state may be a possible explanation of the irregular slope observed upon heating. On cooling down, the material is now crystallised and has a given thermal expansion coefficient smaller than the thermal expansion coefficient of the amorphous state leading to a residual strain (B). Later on, when experiments are reproduced, from instance (point $B$ to point $C$ ), as the material is already crystallised the thermal expansion is no longer modified and the material structure remains similar. Thus, no residual strain is observed. Slopes change observed on cooling from A to B and both on heating and cooling from $B$ to $C$ are not well understood but might be explained but non purely linear relationship between irradiance and temperature. The emissivity of the material is certainly not constant with the temperature.

\section{Resistance behaviour during annealing process}

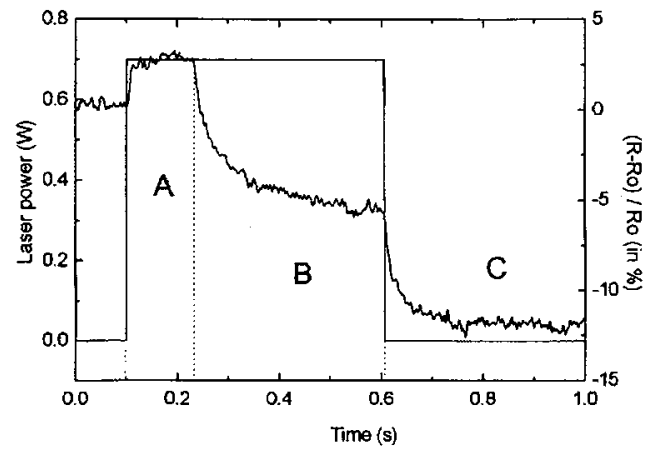

Figure 6: Resistance behaviour of cantilever during the annealing.
During the irradiation process, a resistance change can be clearly observed. The resistance behavior for a laser pulse of about $0.5 \mathrm{~s}$ is shown on figure 6 . The curve can be divided in three regions. The first one (time period A) is assumed to be the material response to the thermal pulse. Then, the resistance decreases (period B) which may be explained by the transformation between the amorphous to the crystal structure. Finally, the laser is turned off and as a consequence of the heat relaxation, the resistance decreases. A relative diminution of about $12 \%$ as been observed in this experiment. The re-
onitoring the annealing process. However, sistance may be the best information source for monitoring the annealing process. However,
further works are needed to correlate the resistance change with the micro-structure induced by the laser like for instance the grain size.

\footnotetext{
1.The structure was laser cut and it is well known that the laser cutting technology introduced a heat affected zone within the material. Cutting parameters were chosen to minimize as much as possible the heat affected zone. This heat affected zone is very difficult to measure and depends on many parameters but it is usually in the order of a few microns
} 


\section{APPLICATIONS OF THE LASMA TECHNOLOGY}

This new technology has been applied for the development of monolithic micro-devices [2]. As mentionned before, the basic idea is to use the laser annealing to induce a local martensitic transformation. Thus, it can be used for inducing a local shape memory effect as well as a local superelastic behavior. Moreover, using different irradiance levels, effects can be modulated throughout the device: for instance, the transformation temperature can be slighty adjusted.

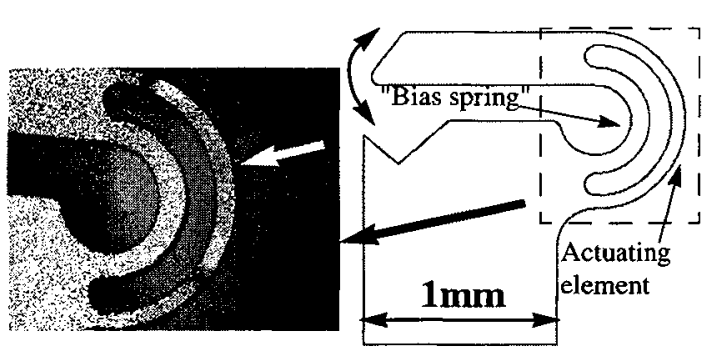

Figure 7: Micro-gripper locally annealed. The annealed zone is clearly visible on the left picture. Considering technological aspects, the LASMA process has been applied on NiTi cold-worked sheet and on sputter-deposited thin film. In the case of a coldworked sheet, the annealing process is used to recrystallise the material and for stress relaxation. In actuators applications, the cold-worked state can be used to provide a biasing force in a monolithic design. A micro-gripper has been developed using this principle [1]. This gripper is shown on figure 7 . When heating up, only the annealed part transforms to austenite pulling down the gripper's arm. On cooling, as the transformed undergoes the reverse transformation, the non-annealed part behaves as a bias spring pushing up the gripper's arm. Therefore a reversible shape change is obtained in a monolithic structure without using any thermomechanical processes.

This technology has also been applied for the development of micro-switch. This micro-device can be seen on figure 8. The device was cut from a 4-micron thick sputter deposited thin film of binary Ni-Ti. After being removed from the substrate, the thin film has a reproducible curvature. This curvature is used to create a motion out of the plane. The local annealing is performed on the middle bar, which expands and bends the structure out of the plane. The biasing effect is given by the non-annealed part of the structure (outside bars). The range motion is in the order $50 \mu \mathrm{m}$. The

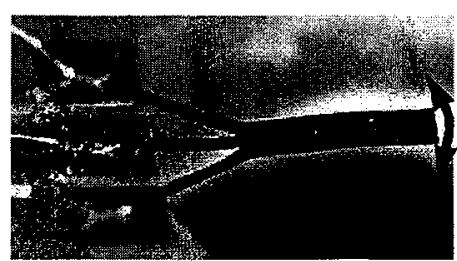

Figure 8: Micro-switch made out Ni-Ti thin film. Length: $1.5 \mathrm{~mm}$ structure is self-prestrained during the annealing process.

\section{CONCLUSION}

The technology of Laser Annealing of Shape Memory Alloys (LASMA) has been introduced. This new process is an efficient tool for building «smart» micro-devices with new capabilities. Future work will be carried out to analyse in more details the expansion phenomena and the related mechanical properties of the locally annealed material as a function of laser parameters. Furthermore, new applications based on this promising technology will be developed.

\section{References}

1. Y. Bellouard, T. Lehnert, J.-E. Bidaux, T. Sidler, R. Clavel, R. Gotthardt, Materials Sciences and Engineering A273-275, pp. 795-798 (1999).

2. Y. Bellouard, T. Lehnert, T. Sidler, R. Gotthardt, R. Clavel in Proceeding of MRS Conference, Boston, 1999, vol. 604 (to be published). 